\title{
Inverse Scattering in a Multipath Environment
}

\author{
Antonio Cuccaro and Raffaele Solimene
}

\author{
Dipartimento di Ingegneria Industriale e dell'Informazione \\ Seconda Universita' degli Studi di Napoli, via Roma 29, Aversa 81031, Italy \\ *antonio.cuccaro@unina2.it
}

\begin{abstract}
In this contribution an inverse scattering problem is addressed in a multipath environment. In particular, multipath is created by known "extra" point-like scatterers (passive elements) expressely deployed between the scene under investigation and the source/measurement domains. Through a back-projection imaging scheme, the role of the passive elements on the achievable performance is shown and compared to the free-space case.
\end{abstract}

\section{Introduction}

The problem of detecting and localising targets can be tackled by adopting a number of different methods [1]. Back-projection, migration algorithms and time reversal are among the most used in literature due to their simplicity of implementation and easiness of understanding [2].

For these methods, it is known that the achievable performance in the reconstructions is basically related to measurement configuration, i.e., the adopted frequency band and the size of the measurement aperture. However, the presence of known in-homogeneities in the background medium can change the things leading to performance enhancement due to the intervening multipath.

Multipath can be a source of artefacts that can corrupt the reconstructions. However, if the in-homogeneous background is someway characterised (for example, because it is artificially created) it can be positively exploited in order to improve the spatial resolution. From a physical point of view, the reason is that in-homogeneities re-radiate the field impinging on them and hence act as a further source of illumination/scattering which virtually enlarges the actual measurement aperture.

A number of papers support previous claim. The effect of a single point-like in-homogeneity located between the sensors and the scene to be diagnosed is studied in [3], whereas the role of a reflecting plane in [4], for inverse source problem, and in [5], for inverse scattering under linearised scattering operators. Under the same assumption, the impact of a reflecting plane located at the bottom of the scattering scene is addressed in the framework of single and multi-frequency case in [6] and [7], respectively. A dielectric periodic structure located in front of the investigation domain is instead considered in [8].

In this contribution, we address a problem similar to the one considered in [14] and perform the reconstruction

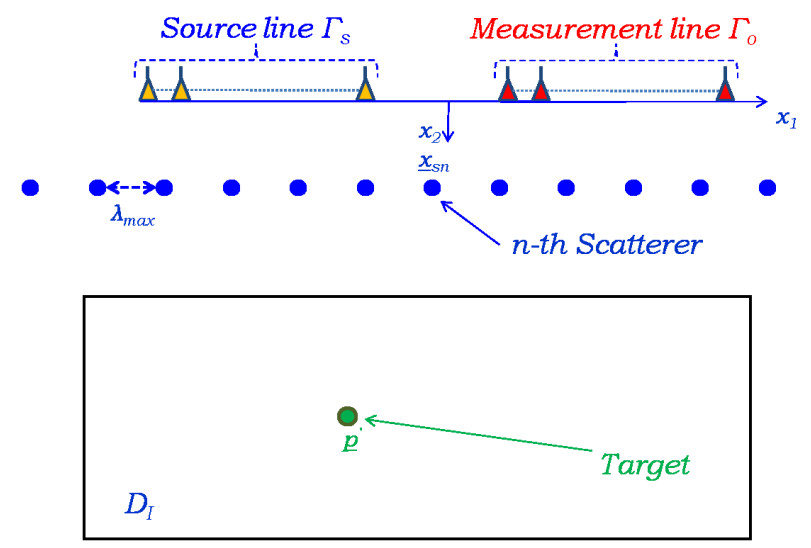

Figure 1: Geometry of interest. The target to be reconstructed is assumed to reside within the investigation domain $D_{I}$. A grid of point-like scatterers (blue circles) is expressely located between the source $\left(\Gamma_{s}\right)$ and observation $\left(\Gamma_{o}\right)$ lines and the target in order to create multipath. The scattered field is collected over the measurement line for all the frequencies within the band $\Omega_{k}$.

via the same inversion back-projection scheme. The goal is to extend the results obtained for inverse source to inverse scattering problems.

It is shown that when a sufficient number of point-like scatterers are deployed in the scene, artefacts they introduce actually superimpose so as to return a focalised contribution. As a consequence, an improvement in the achievable spatial resolution is obtained as compared to the free-space (i.e., when no passive scatterers are present) case.

\section{Mathematical model}

The geometry of interest is depicted in Fig.1 where invariance is assumed along $x_{3}$. The sources are located at $\underline{y}_{s} \in \Gamma_{s}$. They are assumed radiating the signal $S(\omega)$ linearly polarised along the $x_{3}$-axis so that the problem is $2 \mathrm{D}$ and scalar. The scattered field is collected over $\underline{y}_{o} \in \Gamma_{o}$. The target to reconstructed instead resides within the investigation domain $D_{I}$. The scenario also includes an ensemble of $N$ known point-like scatterers located between the source/receiving domains and $D_{I}$. The point-like objects have known spatial positions $\eta_{p}=\left\{\underline{x}_{1}, \underline{x}_{2}, \cdots, \underline{x}_{N}\right\}$ and "strength" $\eta_{m}=\left\{\mu_{1}, \mu_{2}, \cdots, \mu_{N}\right\}$.

Under the Born approximation the scattered field due to 
an object inside the investigation domain $D_{I}$ can be written as

$$
\begin{gathered}
E_{s}\left(k, \underline{y}_{s}, \underline{y}_{O}\right)=k^{2} S(k) \times \\
\int_{D_{I}} G_{N}\left(k, \underline{y}_{O}, \underline{p}\right) \chi(\underline{p}) G_{N}\left(k, \underline{p}, \underline{y}_{s}\right) d \underline{p}
\end{gathered}
$$

where $\chi(p)$ is the contrast function representing the scattering object within the investigation region $D_{I}, k=\omega / c$ is the free-space wavenumber. $G_{N}(\cdot)$ is the Green function pertinent to background medium including the point scatterers.

As the point-like scatterers are by definition much smaller than the employed wavelenght, their scattered field can be considered being proportional to the Green function, $G_{b}(\cdot)$, of the homogeneous medium (free-space). Accordingly, through the Foldy-Lax [9] equations and by further assuming the point scatterers being well-separated (this way multiple scattering between them can be neglected), we can write

$$
\begin{gathered}
G_{N}\left(k, \underline{p}, \underline{y}_{s}\right) \approx G_{b}\left(k, \underline{p}, \underline{y}_{s}\right) \times \\
+\sum_{n=1}^{N} G_{b}\left(k, \underline{p}, \underline{x}_{n}\right) \mu_{n} G_{b}\left(k, \underline{x}_{n}, \underline{y}_{s}\right)=\sum_{n=0}^{N} G_{n}\left(k, \underline{p}, \underline{y}_{s}\right)
\end{gathered}
$$

with $G_{0}\left(k, \underline{p}, \underline{y}_{s}\right)=G_{b}\left(k, \underline{p}, \underline{y}_{s}\right)$ and $G_{n}\left(k, \underline{p}, \underline{y}_{s}\right)=$ $G_{b}\left(k, \underline{p}, \underline{x}_{n}\right) \bar{\mu}_{n} G_{b}\left(k, \underline{x}_{n}, \underline{y}_{s}\right)$.

The latter equation represents our background Green function which obviously incorporates the passive elements' scattering.

The object $\chi($.$) is generally frequency dependent. Such$ a dependence is neglected in our analysis and therefore in operator notation eq. (1) can be written as

$$
\mathcal{G}: \chi(\underline{p}) \in L^{2}\left(D_{I}\right) \rightarrow E\left(k, \underline{y}_{s}, \underline{y}_{o}\right) \in L^{2}\left(\Omega_{k} \times \Gamma_{s} \times \Gamma_{o}\right)
$$

which is a linear integral operator that links $2 \mathrm{D}$ functions. In equation (3) no a priori information related to the source and the field is exploited but the functional spaces they belong to are equipped with usual $L^{2}$ Hilbert structure. $\Omega_{k}$ is the wavenumber frequency band.

\section{Inversion Scheme}

The operator (3) represents the mathematical model to be inverted in order to reconstruct the unknown scatterer. Now, note that through the background Green function (2) the multipath directly influences the scattering operator kernel. Therefore, this phenomenon must effect the reconstruction someway. The main goal here is to foreseen this effect, that is to estimate how extra scatterers act on the achievable performance and to link the latter to the configuration parameters.

In order to invert the model (3) a back-projection scheme is used. This approach actually means processing the scattered field data by the adjoint operator $\mathcal{G}^{\dagger}$
$\mathcal{G}^{\dagger}: E_{s}\left(k, \underline{y}_{s}, \underline{y}_{o}\right) \in L^{2}\left(\Omega_{k} \times \Gamma_{s} \times \Gamma_{o}\right) \rightarrow \tilde{\chi}(\underline{p}) \in L^{2}\left(D_{I}\right)$

of the scatterer operator $\mathcal{G}$. Back-projection algorithms are linear inversion strategy used to achieve the imaging in many areas of applied science, as through-wall imaging (TWI), subsurface imaging (GPR) or in SAR context [2], and in the last two decade even in biomedical applications [10]. Often in literature such methods are addressed in different ways as for example migration [11], time-reversing [12], or back-propagation [13], depending of the scientific contexts. A unified overview of many of those algorithms has been reported in [2]. Actually, performing the inversion through (4) is justified because under certain conditions it is shown that $\mathcal{G}^{\dagger} \mathcal{G}$ can be cast as a pseudo-differential operator [3]. This entails that back-projection allows retrieving the singularities of the unknown (though filtered) in the right location and with the right orientation.

By adopting the expansion (2) in (3), the scattering operator can be rewritten as

$$
\begin{gathered}
(\mathcal{G} \chi)\left(k, \underline{y}_{s}, \underline{y}_{o}\right)=\sum_{n, m=0}^{N}\left(\mathcal{G}_{n m}\right)\left(k, \underline{y}_{s}, \underline{y}_{o}\right) \times \\
=k^{2} S(k) \sum_{n, m=0}^{N} \int_{D_{I}} G_{n}\left(k, \underline{y}_{o}, \underline{p}^{\prime}\right) G_{m}\left(k, \underline{p}, \underline{y}_{s}\right) \chi(\underline{p}) d \underline{p}
\end{gathered}
$$

where with $\mathcal{G}_{00}=G_{0}\left(k, \underline{y}_{o}, \underline{p}\right) G_{0}\left(k, \underline{p}, \underline{y}_{s}\right)$ denotes the free-space path that can be divided in two part: the first one from the source point to the scatterer and the second one from the scatterer to the measurement point. The remaining terms instead account for the paths through the point-like scatterers. A similar expansion can be used for the adjoint operator

$$
\begin{gathered}
\left(\mathcal{G}^{\dagger} E_{s}\right)(\underline{p})=\sum_{l, h=0}^{N}\left(\mathcal{G}_{l h}^{\dagger}\right)\left(k, \underline{y}_{s}, \underline{y}_{o}\right)= \\
\sum_{l, h=0}^{N} \int_{\Omega_{k} \times \Gamma_{s} \times \Gamma_{o}} k^{2} S^{*}(k) \times \\
G_{l}^{*}\left(k, \underline{y}_{o}, \underline{p}\right) G_{h}^{*}\left(k, \underline{p}, \underline{y}_{s}\right) E_{s}\left(k, y_{s}, y_{o}\right) d k d \underline{y}_{s} d \underline{y}_{o}
\end{gathered}
$$

where $*$ represents the conjugate operator. Hence, the reconstruction computed after the adjoint back-projection procedure can be written as

$$
\mathcal{R} \chi(\underline{p})=\int_{D_{I}} \mathcal{K}\left(\underline{p}, \underline{p}^{\prime}\right) \chi(\underline{p}) d \underline{p}
$$

where, obviously, $\mathcal{K}(\cdot)$ is the kernel of the operator $\mathcal{G}^{\dagger} \mathcal{G}$. Actually, $\mathcal{K}(\cdot)$ is the point spread function (PSF) of backprojection inversion scheme. Therefore, it is sufficient to study this term in order to evaluate the affect of the point scatterers on the imaging procedure. By employing (5) and (6), the kernel can be recast as 

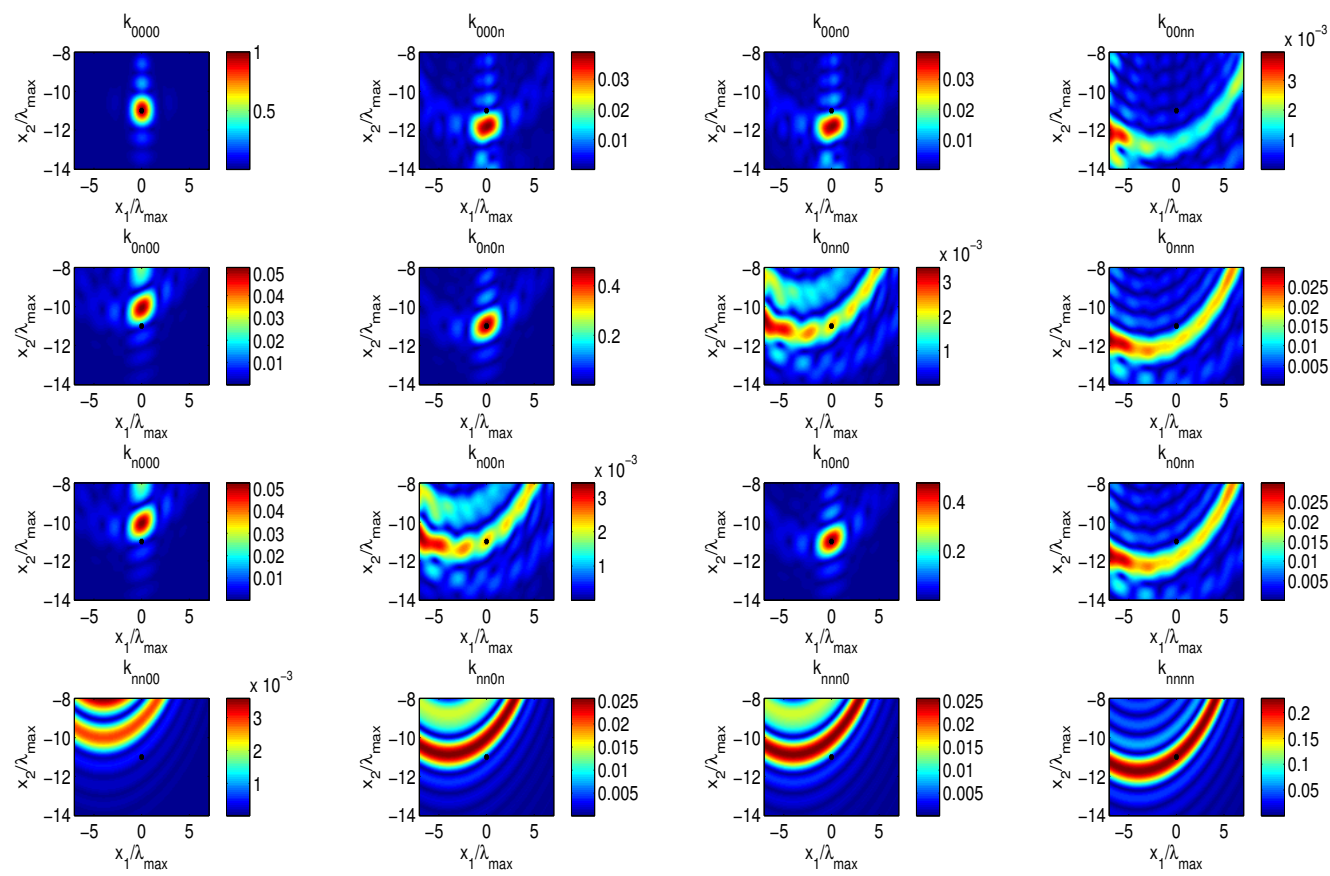

Figure 2: In the panel each single terms is reported when a single scattering point is present. All terms are normalized to $/ \max \left|\mathcal{K}_{0000}\right|$. The point scatterer has $\eta_{1}=6$ it is located at $\left(x_{1}=-4 \lambda_{\max }, x_{2}=-\lambda_{\max }\right)$. The source and measurement line are both uniformly arranged between $[-2 \lambda, 2 \lambda]$ along $x_{1}$ and located at $x_{2}=0$. The target point is denoted by a black bullet.

$$
\begin{aligned}
\mathcal{K}\left(\underline{p}, \underline{p}^{\prime}\right)= & \sum_{n, m, l, h=0}^{N} \int_{\Omega_{k} \times \Gamma_{s} \times \Gamma_{o}} G_{n}^{*}\left(k, \underline{y}_{o}, \underline{p}\right) G_{m}^{*}\left(k, \underline{p}, \underline{y}_{s}\right) \times \\
& G_{l}\left(k, \underline{y}_{o}, \underline{p}^{\prime}\right) G_{h}\left(k, \underline{p}^{\prime}, \underline{y}_{s}\right) d k d \underline{y}_{s} d \underline{y}_{o}
\end{aligned}
$$

where for the sake of simplicity it is assumed $\left|S(k)^{2}\right|=1$ and $k^{4}$ has been considered included in the Green functions.

Each single term can be more conveniently rewritten as

$$
\begin{gathered}
\mathcal{K}_{n m l h}\left(\underline{p}, \underline{p}^{\prime}\right)=\int_{\Omega_{k} \times \Gamma_{s} \times \Gamma_{o}} A_{n m l h}\left(k, \underline{y}_{o}, \underline{y}_{s}, \underline{p}, \underline{p}^{\prime}\right) \times \\
\exp \left[j \Phi_{n m l h}\left(k, \underline{y}_{o}, \underline{y}_{s}, \underline{p}, \underline{p}^{\prime}\right)\right] d k d \underline{y}_{o} d \underline{y}_{s}
\end{gathered}
$$

where the large argument expansion of the Hankel function of second kind and zero order (i.e., the Green function pertinent to a homogeneous medium ) is used. The terms $A_{n m l h}(\cdot)$ take into account the point scatterers strengths, the amplitude cylindrical spreading due to the propagation and in general the source spectrum. The propagation paths instead is embodied in the phase terms $\Phi_{n m l h}(\cdot)$.

Note that the back-projection procedure is implemented without the amplitude correction step proposed in [3]. This is because we are mainly interested in studying how the ensemble of point scatterers contribute in artefact formation and how they can possibly enlarge the spatial retrievable spectrum about the unknown. To this end, phase information is generally enough. The stationary phase method can be employed as in [3] so to approximate each contribution $\mathcal{K}_{n m l h}(\cdot)$ by its asymptotic leading order term. These contributions can be evaluated by solving the following equations

$$
\nabla_{k, \underline{y}_{o}, \underline{y}_{s}} \Phi_{n m l h}=0 \quad \forall n, m, l, h
$$

If equation (10) admit solution only for $\underline{p}=\underline{p}^{\prime}$ then the PSF will be properly focused, otherwise artefacts are expected to appear in the reconstruction.

\section{Imaging}

The reconstruction (7) consists of the summation of $(N+$ $1)^{4}$ terms.

First, we start by considering the case of a single point scatterer so that only 16 terms are relevant. This corresponds to study the subsets of $\mathcal{K}_{n m l h}$ which only involve the subscript 0 and $n \forall n \in(1,2, \cdots, N)$. For those terms, the results shown in [3] can be applied. Here, we omit analytical details and limits to briefly describe such results.

Two types of contribution arise. The first one includes the dominant ones (i.e., the ones coming from the terms $\mathcal{K}_{0000}, \mathcal{K}_{0 n 0 n}$ and $\mathcal{K}_{n 0 n 0}$ ) which have stationary points for all $\left(\underline{y}_{o}, \underline{y}_{s}\right)$ and that correctly focalise in the investigation domain. The second one encompasses the non dominant 

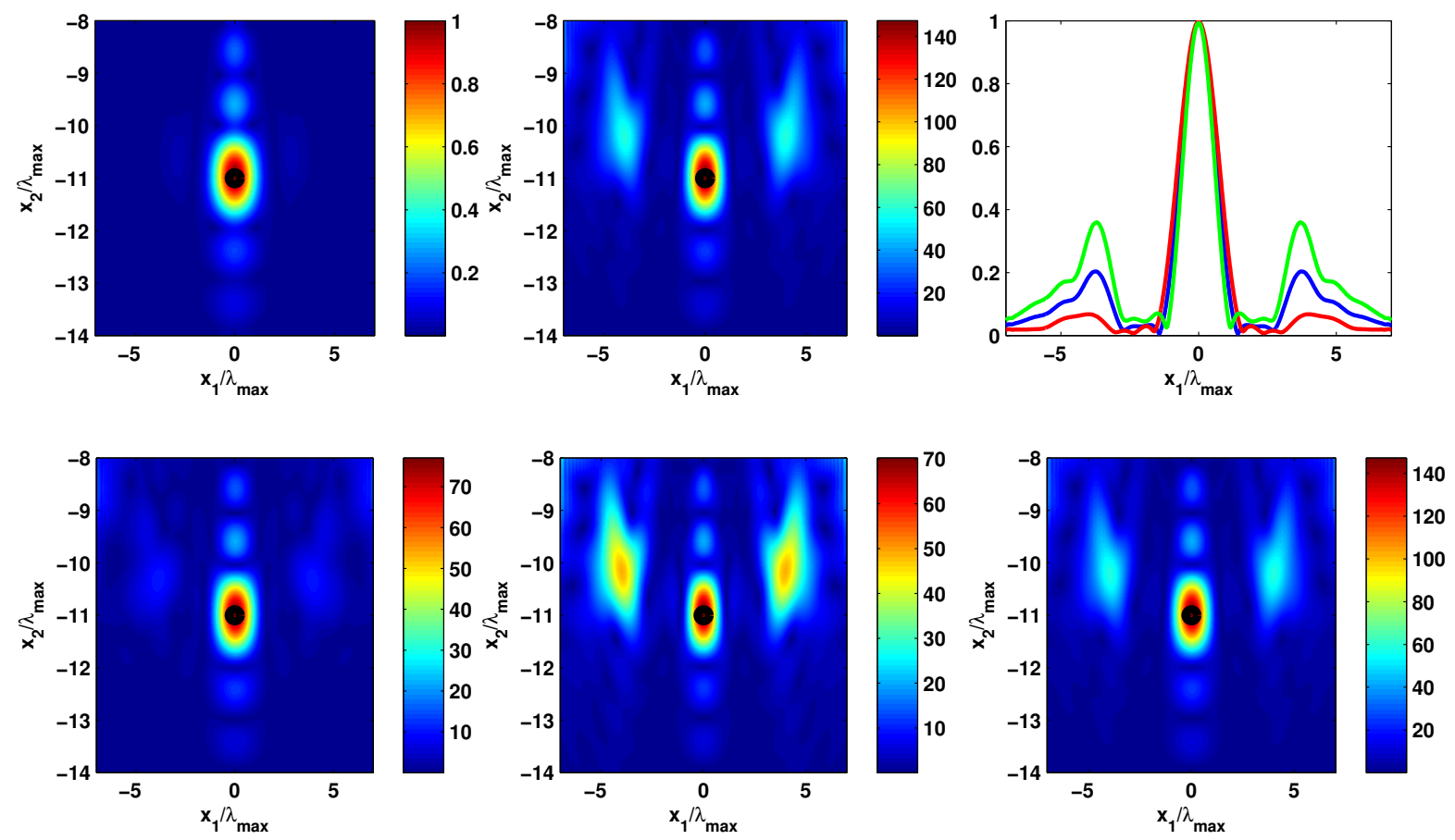

Figure 3: The left and the middle top panels report for comparison purposes $\left|\mathcal{K}_{0000}\right|$ and $|\mathcal{K}|$. The right bottom panel displays the total $|P S F|=\left|P S F_{0}+P S F_{1}+P S F_{2}+P S F 3+P S F_{4}\right|$. Instead, the left and middle bottom panels show the $|P S F| \approx\left|P S F_{0}+P S F_{1}+P S F_{2}\right|$ and $|P S F| \approx\left|P S F_{3}\right|$, respectively. All reconstructions has been normalized to $\left|\mathcal{K}_{0000}\right|$. The right top panel shows the normalised (by their own maxima) cut views passing through the target point (denoted by a black bullet) of $|\mathcal{K}|$ (blue line), $\left|P S F_{0}+P S F_{1}+P S F_{2}\right|$ (red line) and $\left|P S F_{3}\right|$ (green lines). $N=5$ scattering points are uniformly arranged between $[-2 \lambda, 2 \lambda]$ along $x_{1}$ and located at $x_{2}=-\lambda_{\max }$. The target point is denoted by a black bullet.

terms (i.e., coming from $\mathcal{K}_{000 n}, \mathcal{K}_{0 n 00}, \mathcal{K}_{00 n 0}$ and $\mathcal{K}_{n 000}$ ) that have stationary points only when some particular aligning conditions are satisfied. Those second terms provide again a contribution that focalise correctly. However, since these happen only for a single $\left(\underline{y}_{o}, \underline{y}_{s}\right)$, their contribution is lower than the dominant ones. The remaining terms cause potential artefacts when stationary points exist. Fig. 2 shows the reconstructions associated to all the mentioned terms. For example, the terms $\mathcal{K}_{n n n n}$ give rise to circular artefacts. For this reason, the terms $\mathcal{K}_{n n n n}$ have been omitted during the image formation in [3]. Nonetheless, here, we focus right on this kind of terms which, as we are goining to show, are mainly responsible of resolution improvement. However, before proceeding further along this path, the terms $\mathcal{K}_{n m l h}$ not covered by previous discussion must be addressed. To this end, since the point-like scatterers have been assumed not interacting, it is sufficient to consider the kernel terms corresponding to the case of only two point-like scatterers present in the scene. Say $n$ and $m$ their indexes. Now, $3^{4}$ terms are relevant. However, only terms which involve both the $n$ and $m$ subscripts have to be studied as the other ones (i.e., those ones corresponding to the round-trip propagation paths that involve one single point scatterer) have been already covered by previous discussion. By paralleling the same approach as in [3] and in [14], it can be shown that these terms tend to have no stationary points. This is surely true for the point scatterers arrangement (i.e., along a line) that we are considering herein. However, it is worth noting that in general this can depend on the configuration setting (i.e., relative positions between point scatterers and $\Gamma_{o}, \Gamma_{s}$ ). As in the previous case, the dominant contribution turns to come from the terms $\mathcal{K}_{n m n m}$ and $\mathcal{K}_{m n m n}$.

Eventually, as a result of previous discussions the point spread function (8) can be approximated as the sum of five terms

$$
\begin{gathered}
P S F\left(\underline{p}, \underline{p}^{\prime}\right) \approx \mathcal{K}_{0000}\left(\underline{p}, \underline{p}^{\prime}\right)+\sum_{n=1}^{N} \mathcal{K}_{n 0 n 0}\left(\underline{p}, \underline{p}^{\prime}\right)+ \\
+\sum_{n=1}^{N} \mathcal{K}_{0 n 0 n}\left(\underline{p}, \underline{p}^{\prime}\right)+\sum_{n=1}^{N} \mathcal{K}_{n n n n}\left(\underline{p}, \underline{p}^{\prime}\right)+\sum_{n=1}^{N-1} \mathcal{K}_{n m n m}\left(\underline{p}, \underline{p}^{\prime}\right)= \\
=P S F_{0}\left(\underline{p}, \underline{p}^{\prime}\right)+P S F_{1}\left(\underline{p}, \underline{p}^{\prime}\right)+ \\
+P S F_{2}\left(\underline{p}, \underline{p}^{\prime}\right)+P S F_{3}\left(\underline{p}, \underline{p}^{\prime}\right)+P S F_{4}\left(\underline{p}, \underline{p}^{\prime}\right)
\end{gathered}
$$

In the latter equation, $P S F_{0}$ represents the usual term expected from back-projection in free-space which of course focuses on the target. The terms $P S F_{1}$ and $P S F_{2}$ 

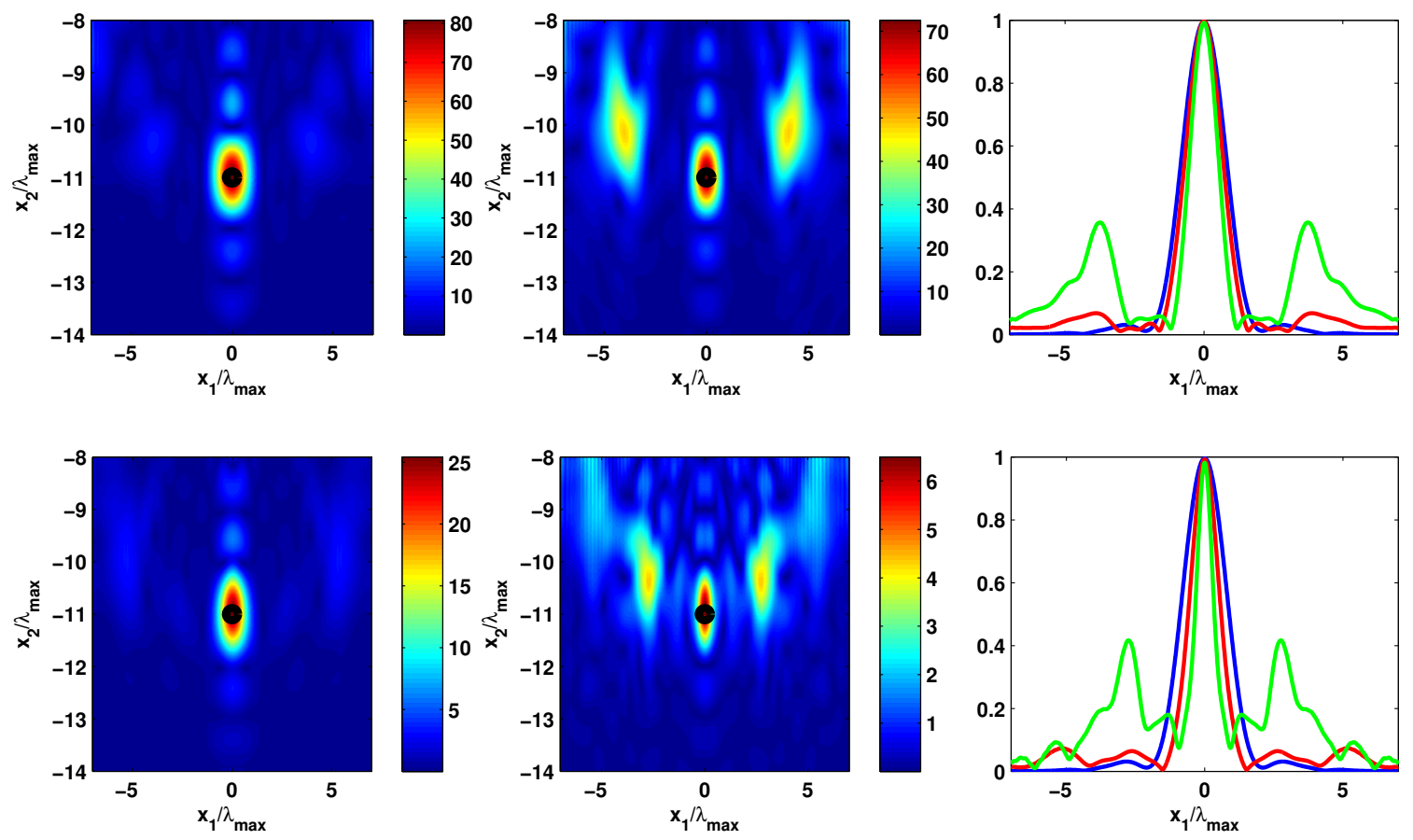

Figure 4: The role of scattering layout: varying the transversal arrangement. The top and the bottom panels refer to $N=11$ scattering points uniformly arranged between $[-5 \lambda, 5 \lambda]$ along $x_{1}$ and located at $x_{2}=-\lambda_{\max }$ and $x_{2}=-4 \lambda_{\max }$, respectively. The left and middle panels display $\left|P S F_{0}+P S F_{1}+P S F_{2}\right| / \max \left|\mathcal{K}_{0000}\right|$ and $\left|P S F_{3}\right| / \max \left|\mathcal{K}_{0000}\right|$, respectively. The right top and low panel show the normalised (by their own maxima) cut views passing through the target point (denoted by a black bullet) of previous reconstructions reported in red and green lines. In blue the cut views of $\left|\mathcal{K}_{0000}\right|$.

are those ones already accounted in [3]. $P S F_{3}$ and $P S F_{4}$ are the terms that we suggest to retain in the reconstruction procedure. Indeed, as mentioned above, each single term in $P S F_{3}$ and $P S F_{4}$ is an artefact that passes through the actual scattering position. Therefore, when they are combined together they give rise to a further focalised contribution. More in detail, by paralleling the same approach as in [14], we got

$$
\begin{gathered}
P S F_{3}\left(\underline{p}, \underline{p}^{\prime}\right)=\sum_{n=1}^{N} b_{3}\left(\underline{p}^{\prime}, \underline{x}_{n}, \Omega_{k}, \Gamma_{o}, \Gamma_{s}\right) \times \\
\hat{\delta}\left(\left|\underline{p}-\underline{x}_{n}\right|-\left|\underline{p}^{\prime}-\underline{x}_{n}\right|\right) \\
P S F_{4}\left(\underline{p}, \underline{p}^{\prime}\right)=\sum_{n=1}^{N-1} b_{4}\left(\underline{p}^{\prime}, \underline{x}_{n}, \underline{x}_{m}, \Omega_{k}, \Gamma_{o}, \Gamma_{s}\right) \times \\
\hat{\delta}\left(\left|\underline{p}-\underline{x}_{n}\right|+\left|\underline{p}-\underline{x}_{m}\right|-\left|\underline{p}^{\prime}-\underline{x}_{n}\right|-\left|\underline{p}^{\prime}-\underline{x}_{n}\right|\right)
\end{gathered}
$$

where the $b_{i}(\cdot)$, with $i=3,4$, are amplitude coefficients whereas $\tilde{\delta}(\cdot)$ is a smoothed version of a delta function due to the filtering introduced by the measurement configuration through the back-projection scheme. Expressions (12) and (13) actually resemble the ruler and compass migration scheme proposed by Hagedoorn [15]. In particular, these terms can be seen as the outcomes of multimonostatic and multistatic (excluding the monostatic data) backprojection schemes, when data are collected at the virtual array (that is not at the actual sources and measurements positions) located at points $\eta_{p}$ (i.e., the point scatterers). This suggests that by suitably arranging the point scatterers in order to have a virtual aperture larger than the actual one, resolution can be improved.

\section{Numerical example}

In this numerical analysis the measurement and transmission lines coincide, i.e., $\Gamma_{o}=\Gamma_{s}=\Gamma$. In particular, they are segments $\left[-2 \lambda_{\max }, 2 \lambda_{\max }\right]$ along the $x_{1}$ axis, and both are located at $x_{2}=0$. The investigation domain is $D_{I}=\left[-7 \lambda_{\max }, 7 \lambda_{\max }\right] \times\left[-8 \lambda_{\max },-14 \lambda_{\max }\right]$ and $\Omega_{k}=[4 \pi, 6 \pi] m^{-1}$. In order to study how the achievable performance is affected by the point scattering object layout, the parameters of the configuration are kept fixed in all the following examples while the scattering points set up is changed. For all the following examples the point scatterers are deployed over a line parallel to $\Gamma$ with a $\lambda_{\max }$ spatial step between two consecutive point scatterers. The latter, in turn, are considered of equal strength $\eta_{m}=6$.

We start the numerical analysis by showing the compar- 

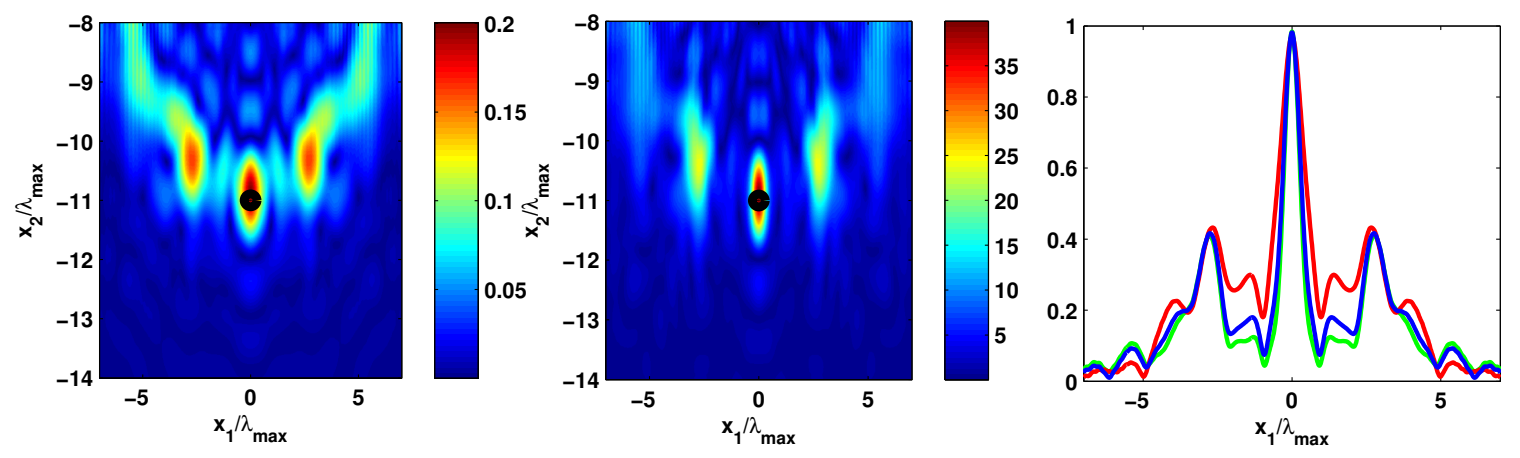

Figure 5: The role of scattering layout: varying the strength of point scatterers. $N=11$ scattering points are uniformly arranged between $[-5 \lambda, 5 \lambda]$ along $x_{1}$ and located at $x_{2}=-4 \lambda_{\max }$. Left and middle panels display $\left|P S F_{3}\right| / \max \left|\mathcal{K}_{0000}\right|$ when $\eta_{m}=2$ and $\eta_{m}=10$, respectively. The right panel shows the normalised (by their own maxima) cut views passing through the target point (denoted by a black bullet) of previous reconstructions reported in red and green lines. In blue the cut views of $\left|P S F_{3}\right| /\left|\mathcal{K}_{0000}\right|$ corresponding to Fig.4 (middle bottom panel).

ison between the total point spread function $\mathcal{K}$ and $\mathcal{K}_{0000}$, which would be obtained without scattering points. The ensemble of point scatterers is located at $x_{2}=-\lambda_{\max }$ and arranged over a segment of length $\left[-2 \lambda_{\max }, 2 \lambda_{\max }\right]$ (i.e., $N=5$ point scatterers are in the scene). By looking at the results reported in Fig. 3, it can be concluded that when the scattering grid is close to $\Gamma$ no significant resolution improvement is observed. This can be explained because for this case the grid and $\Gamma$ have roughly the same "scene view", that is they practically subtend the same observation cone. This means that the spatial wavenumber band is unchanged by the grid presence and therefore the transverse resolution does not increase. In order to appreciated the goodness of the point spread function approximation in the same figure is depicted the reconstruction obtained by using equation (11) (the same result is obtained if term $\mathrm{PSF}_{4}$ is omitted). As can be seen, it is very similar to $\mathcal{K}$ (Fig. 3, middle top panel). Accordingly, we conclude that by using the approximated imaging procedure not only it is reduced the computational load (faster computationally) than the traditional back-projection, but it provides similar results to the latter. In the same figure is shown the reconstruction (left bottom panel) obtained when in the inversion back-projection strategy only the first three terms are employed. It is similar (except for some artefact) to result depicted in the middle bottom panel, where instead the imaging procedure is carried out by means only $P S F_{3}$ term. This observation suggests to adopt in the inversion scheme only the latter term since it allows to further reduce the computational burden. Hereafter the inversion backprojection scheme is achieved through or only the first three contribute $P S F_{0}, P S F_{1}, P S F_{2}$ or just the last $P S F_{3}$.

The second example is shown in Fig. 4. Here, the passive grid is arranged over the segment $\left[-5 \lambda_{\max }, 5 \lambda_{\max }\right]$ (i.e., $N=11$ ) and it is first located at $x_{2}=-\lambda_{\max }$ (Fig. 4 , top panels) and then moved at $x_{2}=-7 \lambda_{\max }$ (Fig. 4, bottom panels). As can be seen, when the imaging procedure is implemented by adopting the first three terms of equation (11), the transverse resolution is improved slightly in both scenarios. However, this improvement becomes more pronounced when the back-projection is carried through only the term $P S F_{3}$, particularly when the grid is closer to $D_{I}$ (Fig. 4, middle bottom panel). These results are consistent with previous discussion. Indeed, in both the previous case the grid subtend a larger observation cone (with respect the actual measurement aperture) which means a larger spatial wavenumber band and hence a better transverse resolution. However, this improvement is less marked when the grid is close to $\Gamma$. It can be shown that this is due to the coefficient $b_{i}(\cdot)$ which suffer from of a sort of tapering (magnitude decays towards the edges of the grid) which reduce the virtually aperture relevant in the reconstructions. Moreover, it is worth noting that in many applications, the resolution of the imaging algorithm is defined at $-3 \mathrm{~dB}$ with respect to the maximum amplitude of the reconstruction (i.e., only within this interval the amplitude of the reconstruction is preserved). Therefore, although some artefact appears in the reconstruction (Fig. 4, middle top and bottom panels), it can be said that an improvement in resolution occurs. This can be appreciated by cut views through the target position reported in the same figure. Finally, it is noted the reconstruction amplitude of Fig.4 (left and middle top panels) slightly increases (as compared to the case of Fig. 3, left and middle bottom panel). This is because the number of grid elements is increases as well.

The last example shown in Fig. 5 concerns the case where the strength of scatterers is changed. In particular, by choosing the same configuration as in Fig. 4 (middle bottom panel), the strength of scatterers is chosen first equal to $\eta_{m}=2$ and then increased at $\eta_{m}=10$. By looking at the figures, it can be appreciated the different reconstruction amplitudes, which obviously is a consequence of the adopted different strengths. As far as resolution is concerned, it can be seen that with weak point scatterers the 
resolution improvement achieved when $\eta_{m}=6$ (Fig. 4, middle panel) disappears. Otherwise, beyond a threshold value of $\eta$ it is preserved and keep unchanged. Therefore, further increasing $\eta$ entails only an increase of maximum amplitude of the reconstruction. The cut views depicted in Fig. 5 (right panel) shown clearly this aspect.

\section{Conclusions}

In this paper an inverse scattering problem in a multipath environment caused by an ensemble of scattering points has been dealt with for a 2D scalar setting through a backprojection inversion method. By approximating the PSF thanks to the stationary phase method, the contribution to the reconstruction due to the scattering grid has been evidenced. In particular, it has been shown that the grid can allow to obtain a finer resolution (compared to free-space) and that the mainly responsible of this resolution improvement is the term $P S F_{3}$. It remember that the latter is considered as artefact in [3] and therefore it is discarded in image formation procedure proposed there.

Finally, it is highlighted that with our proposed procedure (back-projection through only $P S F_{3}$ ) two important aspect are combined: an improvement of the resolution achievable thanks to extra point scatterers and a faster imaging procedure compared to the traditional one.

As future developments, we plan to extend to study to the inverse scattering problem to a 3D dyadic case.

\section{Acknowledgement}

This work was supported by the Italian Ministry of Uni-

versity and Research through the FIRB initiative under the project MICENEA (RBFR12A7CD).

\section{References}

[1] I. Catapano, A. Randazzo, E. Slob and R. Solimene, GPR imaging via qualitative and quantitative approaches, in Civil Engineering Applications of Ground Penetrating Radar, Springer International Publishing, pp. 239-280, 2015.

[2] R. Solimene, I. Catapano, G. Gennarelli, A. Cuccaro, A. Dell'Aversano and F. Soldovieri, SAR imaging algorithms and some unconventional applications: a unified mathematical overview, IEEE Sign. Process. Magaz. 31: 90-98, 2014.

[3] M. Cheney and R.J. Bonneau, Imaging that exploits multipath scattering from point scatterers, Inverse Probl. 20: 1691-1711, 2004.

[4] R. Solimene, M.A. Maisto, R. Pierri, Inverse source in the presence of a reflecting plane for the strip case, J. Opt. Soc. Am. A 31: 2814-2820, 2014.

[5] R. Solimene, M.A. Maisto, R.-Pierri, Inverse scattering in presence of a reflecting plane, J. Opt. 18: 025603, 2016.
[6] A.J. Devaney and M. Dennison, Inverse scattering in inhomogeneous background media, Inverse Probl. 19: 855-870, 2003.

[7] M.L. Dennison and A.J. Devaney, Inverse scattering in inhomogeneous background media: II. Multifrequency case and SVD, Inverse Probl. 20: 13071324,2004

[8] P.C. Chang, R.J. Burkholder, and J.L. Volakis, ModelCorrected Microwave Imaging through Periodic Wall Structures, Int. J. Antenn. Prop. ID 948365, 2012.

[9] L.L. Foldy, The multiple scattering of waves, Phys. Rev. 67: 107-119, 1945.

[10] H. Lim, N. Nhung, E. Li, and N. Thang, Confocal microwave imaging for breast cancer detection: delaymultiply- and-sum image reconstruction algorithm, IEEE Trans. Biomed. Eng. 6: 1697-1704, 2008.

[11] G. Gilmore, I. Jeffry, and J.Lo Vetri, Derivation and comparison of SAR and frequency-wavenumber migration within a common inverse scalar wave problem formulation, IEEE Trans. Geosci. Remote Sensing 44: 1454-1461, 2006.

[12] D. Cassereau and M. Fink, Time-reversal of ultrasonic fields part III: Theory of the closed time-reversal cavity, IEEE Trans. Ultrason. Ferroelectr, Freq. Control 39: 579-592, 1992.

[13] C. Esmersoy and D. Miller, Backprojection versus backpropagation in multidimensional linearized inversion, Geophysics 54: 921-926, 1989.

[14] R. Solimene, A. Cuccarao and R. Pierri, BackProjection Source Reconstruction in Presence of Point Scatterers, J. Opt. 18: 065606, 2016.

[15] J.G. Hagedoorn, "A process of seismic reflection interpretation," Geophys. Prosp. 2: 85-127, 1954. 